\title{
Characterizing the Shape of Anatomical Structures With Poisson's Equation
}

\author{
Haissam Haidar[Member, IEEE], \\ Department of Neurology, Children's Hospital and Harvard Medical School, Boston, MA 02115 \\ USA
}

\section{Sylvain Bouix[Member, IEEE],}

Psychiatry Neuroimaging Laboratory, Department of Psychiatry, Brigham and Women's Hospital and Harvard Medical School, Boston, MA 02114 USA (sylvain@bwh.harvard.edu)

\begin{abstract}
James J. Levitt,
Psychiatry Neuroimaging Laboratory, Department of Psychiatry, Brigham and Women's Hospital and Harvard Medical School, Boston, MA 02114 USA, and also with the Clinical Neuroscience Division, Laboratory of Neuroscience, Boston VA Healthcare System, Brockton Division, Department of Psychiatry, Harvard Medical School, Boston, MA 02301 USA
\end{abstract}

\section{Robert W. McCarley,}

Clinical Neuroscience Division, Laboratory of Neuroscience, Boston VA Healthcare System, Brockton Division, Department of Psychiatry, Harvard Medical School, Boston, MA 02215 USA

Martha E. Shenton, and

Psychiatry Neuroimaging Laboratory, Department of Psychiatry, Brigham and Women's Hospital and Harvard Medical School, Boston, MA 02114 USA, and also with the Clinical Neuroscience Division, Laboratory of Neuroscience, Boston VA Healthcare System, Brockton Division, Department of Psychiatry, Harvard Medical School, Boston, MA 02301 USA

\author{
Janet S. Soul \\ Department of Neurology, Children's Hospital and Harvard Medical School, Boston, MA 02115 \\ USA
}

\section{Abstract}

\begin{abstract}
Poisson's equation, a fundamental partial differential equation in classical physics, has a number of properties that are interesting for shape analysis. In particular, the equipotential sets of the solution graph become smoother as the potential increases. We use the displacement map, the length of the streamlines formed by the gradient field of the solution, to measure the "complexity" (or smoothness) of the equipotential sets, and study its behavior as the potential increases. We believe that this function complexity $=f$ (potential), which we call the shape characteristic, is a very natural way to express shape. Robust algorithms are presented to compute the solution to Poisson's equation, the displacement map, and the shape characteristic. We first illustrate our technique on two-dimensional synthetic examples and natural silhouettes. We then perform two shape analysis studies on three-dimensional neuroanatomical data extracted from magnetic resonance (MR) images of the brain. In the first study, we investigate changes in the caudate nucleus in Schizotypal Personality Disorder (SPD) and confirm previously published results on this structure [1]. In the second study, we present a data set of caudate nuclei of premature infants
\end{abstract}

\footnotetext{
(C) 2006 IEEE

Correspondence to: Sylvain Bouix.
} 
with asymmetric white matter injury. Our method shows structural shape differences that volumetric measurements were unable to detect.

\section{Index Terms}

Brain morphometry; caudate nucleus; Poisson's equation; shape analysis

\section{INTRODUCTION}

In both clinical and research contexts, quantitative models for the three-dimensional (3-D) shape of anatomical structures might allow an alternative approach for the statistical analysis of their distinct characteristics. In fact, there has been significant progress in the development of algorithms for use in computational anatomy, as described by several recent articles in [2]. A first class of methods uses a global feature vector, e.g., determined by spherical harmonics or invariant moment representations [3], [4], and attempts to discriminate between classes of shapes using clustering techniques. Such methods are usually numerically stable and allow for the computation of relevant statistics. However, the resulting feature vector is rarely intuitive, hence an interpretation of the results in terms of anatomical changes can be difficult. A second class of methods is based on a representation of an object's surface or interior, along with a study of the mechanical deformations required to transform one object into another [5]-[8]. This popular class of techniques is very intuitive, but relies heavily on the use of nonlinear registration techniques to establish one to one correspondences between subjects. There are two main issues with these techniques. First, the hypothesis that a one to one correspondence must be established is not obvious. Objects under study must have very similar shapes in order for this hypothesis to make sense. For example, it is not clear how such methods would perform if they were confronted with the task of comparing hands with four fingers versus hands with five fingers. An interesting discussion of this problem has been argued in the context of Voxel Based Morphometry [9], [10]. Second, automatic nonlinear registration is a challenging problem and the accuracy and robustness of current algorithms is difficult to validate [11]. The calculation of statistical significance from the recovered deformation fields also poses a challenge as the number of tests is usually much larger than the number of subjects under study [12], [13]. A third class makes use of medial representations which provide information on an object's reflective symmetries. Unfortunately, the one to one correspondence assumption is still necessary whether it is done through registration [14] or modeling [15]. In medical studies, different classes of methods are often combined in order to obtain intuitive and statistically significant results (see, for example, [13]).

The main focus of this paper is to try to detect shape differences of anatomical structures between different populations (e.g., the shape of the hippocampus in Schizophrenics versus Depressives versus Normal controls). Given $n$ binary maps (representing a specific anatomical structure) divided into $k$ groups, we want to know if the shape of the structure is statistically significantly different across groups. In order to accomplish such a task, one first has to settle on a mathematical model for shape and the means to compute it robustly. Second, one has to derive a computational framework in which objects can be compared. This involves the definition of a metric and a notion of correspondence between models. Finally, one has to be able to extract statistics that are sensitive enough to detect subtle changes between populations yet robust enough to avoid common statistical errors.

We propose a novel approach to shape analysis based on a well-known partial differential equation (PDE), Poisson's equation 


$$
\nabla^{2} \phi=-1
$$

This equation, which is best known in electrostatics, has very interesting properties for the study of shape. First, we model shape by the solution to Poisson's equation over the interior of the object with a constant Dirichlet boundary condition. The solution, often called the potential function, is smooth, has a finite set of local maxima, and is invariant to rigid transformations. We then study the smoothness of the level sets of the potential function by designing a feature vector expressing for each equipotential level set a simple smoothness measure. The feature vector is used to compare different objects and allows for robust statistics through randomization tests. This method is in essence a global feature vector based technique, although we believe the vector contains information that is sufficiently intuitive to allow for an anatomical interpretation of the results. Our technique is conceptually related to the work of Gorelick et al., who recently investigated the potential of Poisson's equation to describe and classify two-dimensional (2-D) objects [16]. The method we propose was developed independently and has a number of key differences which will be explained in Section II-B.

We illustrate the power of this representation in 2-D with both simple synthetic shapes and silhouettes of natural objects. We also evaluate our shape model on medical data, by performing two studies in which we show differences in the shape of the caudate nucleus between subject affected with an illness and normal controls. The first study analyzes a group of subjects diagnosed with Schizotypal Personality Disorder (SPD) and age matched normal controls. A significant difference in the shape of the right caudate was found between SPD subjects and normal controls $(p=0.023)$, replicating a result previously found in the same subjects but using an entirely different shape analysis approach [1]. The second study investigates the effect of unilateral white matter injury on the shape of the caudate nucleus of premature infants. Again, a significant difference was found between the shape of the caudate on the injured side compared with the normal side $(p=0.031)$ whereas no difference between sides was found in the shape of the caudate nucleus in premature infants with no brain injury. More importantly, even though we detected no difference in size between the caudate nucleus on the unaffected side of infants with white matter injury and the caudate of normal infants, we found shape differences $(p=0.033)$.

\section{METHODS}

\section{A. Poisson's Equation}

1) Definition and Properties-Poisson's equation is a second-order elliptic partial differential equation widely used to solve problems in gravitational, electric and electromagnetic fields and in other applications from mathematical physics. For example, in order to find the electric potential $\phi$ (also known as voltage) in an electric field, one has to solve the following Poisson equation:

$$
\nabla^{2} \phi=-\frac{\rho}{\varepsilon_{0}}
$$

where $\rho$ is the charge density and $\varepsilon_{0}$ is the permittivity of free space.

In this paper, we use a very simple version of this equation in order to model shape. First, we consider that our domain of interest is made of equally distributed positive unit charges. Second, we assume that the potential on the boundary $C_{0}$ of the domain is known (Dirichlet 
boundary condition) and constant. In order to find the potential field over the entire domain, one has to solve the following canonical form of Poisson's equation:

$$
\nabla^{2} \phi=-1, \quad \phi\left(C_{0}\right)=k
$$

where $k$ is a constant.

Functions $\phi$ satisfying Poisson's equation are often called potential functions. They have many mathematical properties related to the geometry of the boundary of the domain. First, the solution to Poisson's equation is unique and smooth, and in the special case of Dirichlet conditions the potential function has a finite set of local maxima. A unique streamline can be drawn from each point on the boundary to each local maximum by following the gradient field $\nabla \phi$ (Fig. 1). In electrostatics, the length of the streamline is referred to as the electric displacement and can be plotted as a function over the domain. The shape and length of the streamlines are independent of the constant value of the potential on the boundary and are closely related to the shape of the domain. For example, an interesting approach to partition a shape into different parts would be to separate sets of boundary points associated with different local maxima. The function $\phi$ can also be seen as an embedding of equipotential level curves

$$
\phi(C(t))=t .
$$

Interesting properties of the level curves $C$ can be computed from $\phi$. The normal to the curve is $\hat{\mathbf{N}}=\nabla \phi /\|\nabla \phi\|$. The curvature is given by $k=\nabla .(\nabla \phi /\|\nabla \phi\|)$.

Expressing the shape of an object by a function over its interior is not new. For example, the solution to the Laplace equation, $\nabla^{2} \phi=0$, has been used for measuring thickness between two surfaces [17], [18]. Also, a large body of work has been concentrating on the distance transform, the solution of the following Eikonal equation $\|\nabla \phi\|=1$ [12], [19], [20]. One disadvantage of the distance function is that it is not smooth and there are locations in the interior of the object for which $\nabla \phi$ is not defined. These discontinuities are very interesting to study, as they define the location of the medial axis [21], a shape representation central to many imaging studies [14], [15], [22], [23]. On the other hand, as Gorelick et al. noted, one very interesting property of the level curves of the solution to Poisson's equation is that they become smoother and smoother as the potential increases, eventually collapsing to one of the maxima of $\phi[16]$. The main idea of this paper is to use this property to characterize shape, by measuring the "smoothness" of the equipotential level sets as they approach a maximum and express it as a function "smoothness $=f$ (potential)." This function can then be used as a feature vector for shape analysis.

2) Numerical Solutions-Our shape analysis technique relies on the computation of two functions. First, we need to obtain the potential $\phi$ defined by (1). Second, we are interested in computing the displacement function in a robust and efficient manner.

a) Poisson's potential function: Computational methods to solve elliptic partial differential equations are standard [24]-[26]. In this paper, we implemented an efficient algorithm for finding the solution to this equation on a 3-D voxel grid based on our previous work for solving the Laplace equation [27]. The algorithm is based on finite difference approximations, which are discrete techniques wherein the domain of interest is represented by a set of points or nodes and information between these points is commonly obtained using Taylor series expansions. In our application, we use a second-order finite difference approximation to solve Poisson's equation. The algorithm consists of representing the 3-D 
potential function as a simple linear regression of three orthogonal 2-D potential functions, each representing the solution of a Poisson equation in 2-D. For a more detailed description of this algorithm, we refer the reader to [27].

b) Displacement maps: Computing the displacement $D$ at each point $\mathbf{x}$ in the domain, is equivalent to computing the geodesic distance from the boundary to $\mathbf{x}$. A streamlining algorithm can be used to calculate the displacement at voxel $\mathbf{x}$ by integrating the Euclidean distance between consecutive voxels along the streamlines connecting the boundary to the local maxima. Unfortunately, this technique will have trouble converging as the automatic detection of local maxima can be particularly difficult in the discrete domain of a 3-D grid.

A more elegant method is the one proposed by Yezzi and Prince for the measurement of thickness [18]. They define thickness between two surfaces as the summation of the length of two different streamlines, one that follows the gradient field of some potential function to the inner surface (downwind) and another that goes against the gradient field to reach the outer boundary (upwind). They derive a robust and efficient iterative algorithm for computing these lengths which can be directly applied to compute our displacement.

In our work, we only have one surface and the information flows inwards, we thus need to apply an upwind scheme to compute the displacement. Such scheme has no problem converging to the boundary of the structure and allows for the computation of the displacement maps without the explicit detection of potential multiple maxima.

Let $T_{X}, T_{y}$, and $T_{Z}$ be the $x, y$, and $z$ components of the normalized gradient $\nabla \phi /\|\nabla \phi\|$ of the potential function. The displacement can be computed by the following recurrence:

$$
\begin{gathered}
\forall(x, y, z), \quad D_{0}(x, y, z)=0 . \\
D_{i+1}(x, y, z)=\frac{1+\left|T_{x}\right| D_{i}(x+\Delta x, y, z)}{\left|T_{x}\right|+\left|T_{y}\right|+\left|T_{z}\right|} \\
+\frac{\left|T_{y}\right| D_{i}(x, y+\Delta y, z)+\left|T_{z}\right| D_{i}(x, y, z+\Delta z)}{\left|T_{x}\right|+\left|T_{y}\right|+\left|T_{z}\right|} .
\end{gathered}
$$

The displacement information flows in the forward direction of the gradient field $\nabla \phi /\|\nabla \phi\|$ (downwind). It is important to define an upwind differencing scheme so that the computation of $D$ is accurate and handles multiple maxima in $\phi$. Thus, $\Delta x, \Delta y$, and $\Delta z$ are defined in the backward direction of the normalized gradient $\nabla \phi /\|\nabla \phi\|$ :

$$
\begin{aligned}
& \Delta x= \begin{cases}-1, & T_{x}>0 \\
1, & T_{x}<0\end{cases} \\
& \Delta y= \begin{cases}-1, & T_{y}>0 \\
1, & T_{y}<0\end{cases} \\
& \Delta z=\left\{\begin{array}{ll}
-1, & T_{z}>0 \\
1, & T_{z}<0
\end{array} .\right.
\end{aligned}
$$

The process is stopped when $\left|D_{i+1}-D_{i}\right|<\epsilon$, a predefined convergence threshold. As suggested by Yezzi and Prince, there are different traversal strategies to update the displacement grid. In our framework, we used the "Gauss-Seidel" traversal. We refer the reader to [18] for more details on computing $D$ efficiently. Fig. 2 and Fig. 4 show a number of synthetic and natural planar objects for which the potential function $\phi$ and the displacement $D$ have been computed. Note that in Fig. 4 many of the structures have 
potential functions with more than one local maxima, which our algorithm is able to handle properly.

\section{B. Shape Characteristic}

We believe the Poisson equation can open the door to many types of shape measurements. Its smooth nature and uniqueness can allow for very robust computations, and the discrete finite number of local maxima suggests its use for partitioning shape into parts. In 2-D, Gorelick et al. have derived a number of very interesting measures from the potential $\phi$ that can help detect corners, extract different parts of the object or describe a skeleton-like structure. They combined a number of these measures in a feature vector which was then used for shape classification and matching [16].

Our application is 3-D and our aims are different; thus, a different feature vector was designed. Our primary objective is to establish a framework to differentiate the shape of an anatomical structure of interest between two populations. From a numerical standpoint, we need a feature vector that is easy to compute and of reasonable size so that the statistical analysis is not impaired by an unmanageable number of tests. From an anatomical standpoint, we wish to obtain information that can be easily interpreted in terms of anatomical changes and biological hypotheses about the populations under study. There is always a trade off between precision (detection of subtle changes) and easy interpretation on one hand, and robustness of the computation on the other hand. One has to find a good compromise, our approach emphasizes on robustness while trying to preserve some intuition on the possible underlying anatomical differences.

As we have noted in Section II-A, the equipotential surfaces become smoother as the potential increases in the domain. Our assumption is that the dynamics of change of the equipotential surfaces as they reach maximal potential is closely related to the geometry of the boundary and can help differentiate different types of objects. To model this process, we introduce a function which evaluates the amount of complexity the equipotential surfaces lose as they approach a maximum point. We measure complexity by the coefficient of variance of the displacement along the current equipotential. We recognize that other measures, such as the surface's mean curvature (or curve curvature in 2-D), could be used, but have found our measurement to be quite stable and applicable to arbitrary dimensions. This function is the basis of our analysis. It can be viewed as a feature vector that can be easily incorporated in a robust statistical framework. The precise nature and location of the shape change cannot be inferred from this function, but its interpretation in terms of anatomical phenomena is still relatively intuitive. For example, a quick drop of complexity at a low potential is probably due to boundary noise or bumps. An overall, slower slope would mean a more elongated shape.

We now turn to the formal mathematical definition of our complexity(potential) function. In order to compare different objects, we decided not to use the absolute potential, but to normalize it between 0 and 1 . This notion of a normalized drop of potential $E$ at an equipotential surface $S_{i}$ is expressed as

$$
E=\frac{\left(\phi_{0}-\phi_{i}\right)}{\left(\phi_{0}-\phi_{\max }\right)}
$$

$\phi_{0}, \phi_{\max }$, and $\phi_{i}$ are the potentials on the boundary, at the global maximum point, and on the current equipotential surface, respectively. We then introduce $v$, the coefficient of variance of the displacement along the current equipotential surface $S_{i}$ 


$$
v(E)=\frac{\operatorname{stdev}\left(D\left(S_{i}\right)\right)}{\operatorname{mean}\left(D\left(S_{i}\right)\right)} .
$$

The function $v(E)$, which we call the shape characteristic, has some interesting properties. By design, it is independent of the size of the object and of the initial potential on the outer boundary. Because the equipotential surfaces get smoother as the potential increases, the curve $v(E)$ monotically decreases and reaches zero when $E=1$. We believe that the rate at which $v$ decreases is characteristic of the shape of an object. For example, a 3-D sphere will have $v(E)=0$ for any $E$. Similarly, $v(E)$ of a cube should be quite different from the one of an ellipsoid. In the next section, we present the shape characteristics for a number of canonical 2-D objects and natural silhouettes. We suggest it could be used for object classification.

\section{2-D Examples}

Fig. 3 is a plot of the shape characteristics of different synthetic images. Even though we only have four objects, the shape characteristic curves are clearly distinct. As expected for the circle, $v(E)=0$. As the energy level increases, it takes relatively little effort to "round" the corners of the square, whereas the ellipse keeps its smooth shape for longer until it "collapses" to a circle.

For natural shapes, we had nine objects equally partitioned in three different classes: hands, horses, and reptiles. We computed the solution to Poisson's equation and calculated the displacement for each object, as shown in Fig. 4. Their respective shape characteristics were derived and are displayed in Fig. 5. First, observe that the shapes are in many ways similar, they all have a central part from which emerge a few "limbs." Nevertheless, the shape characteristic is able to capture the subtle differences between the classes and one can clearly observe three different profiles for the curves corresponding to the three different classes of objects under study. We have not done any 2-D classification experiments, but it would be interesting to train a standard classifier such as a neural network or support vector machine to test the discriminative power of this simple curve [28]. Our intuition, $p$ from Fig. 3 and Fig. 5, is that the shape characteristic may be quite appropriate to discriminate between different classes of shape, but not precise enough to distinguish shapes that are only slightly different (e.g., different hands). Nevertheless, as we will show in the next sections, our technique is sufficiently powerful to detect subtle group differences between patients and normal controls in the shape of a neuroanatomical structure.

\section{Statistical Analysis Framework}

Given two populations $A$ and $B$, we wish to know if the shape of the subjects in $A$ is different than the shape of the subjects in $B$. Our shape characteristic is clearly nonlinear and no assumption of normal or Gaussian distribution of the data can safely be made, thus, we chose nonparametric permutation tests as the basis of our statistical analysis [29]. Permutation tests have become increasingly popular in the medical imaging community as they assume very little about the distribution of the data and allow for robust and elegant handling of the multiple comparisons problem [13], [30], [31].

Our null hypothesis is that there is no difference between the mean shape characteristic of population $A$ of size $a$ and $B$ of size $b$. We test this null hypothesis as follows.

1. Discretize the shape characteristics at the following normalized potential levels: $0.1,0.2,0.3, \ldots, 0.9,1$. 
2. Calculate the difference $D$ between the mean discretized shape characteristics of the two groups as the $L_{2}$ norm of the difference vector between the mean characteristics.

3. Set $D_{0}=D$ the true difference between group A and B.

4. Put all shapes from groups $\mathrm{A}$ and $\mathrm{B}$ into a common basket, and create two new sets, $A_{i}$ of size $a$, and $B_{i}$ of size $b$, by randomly selecting shapes from the basket.

5. Repeat steps 2,3 , and $5 N$ times. Store the mean difference $D_{i}$ at each iteration.

6. Calculate the number $M$ of selections for which $D_{i}>D_{0}$.

7. The $p$-value for the significance of shape difference between groups $A$ and $B$ is then given as: $p=M / N$.

Fig. 8 illustrates how distributions are created using the permutation process for the SPD data. Note the non parametric nature of the distributions especially on the right anterior caudate. We now have all the necessary tools to analyze medical data to detect possible shape changes in a neuroanatomical structure between two populations.

\section{ANALYSIS OF MEDICAL DATA}

\section{A. Schizotypal Personality Disorder}

The caudate nucleus is an essential part in the cognitive circuitry connecting the frontal lobe to subcortical structures of the brain. Pathology in any of the core components of this circuitry may result in a neuropsychiatric condition such as schizophrenia [32], [33] or SPD. Previous studies have shown volumetric and shape differences of the caudate between normal controls and SPD subjects [1], [34]. In this section, we propose to test our methodology by applying our shape analysis method to the data used in [1] and [34] and observe if the results are similar to our previous studies.

1) Data-Fifteen right-handed male subjects with SPD with no previous neuroleptic exposure and fourteen normal comparisons subjects (NC), underwent magnetic resonance imaging (MRI) scanning. Subjects were matched for parental socioeconomic status, handedness, and gender. MRI images were acquired with a 1.5T GE scanner, using a SPoiled Gradient Recalled (SPGR) sequence yielding a $(256 \times 256 \times 124)$ volume with $(0.9375 \times 0.9375 \times 1.5 \mathrm{~mm})$ voxel dimensions. The scans were acquired coronally. The caudate nuclei were drawn manually and separated by an Anterior/Posterior boundary (see Fig. 6). Details of the segmentation procedure can be found in [34]. The subjects and manual segmentations studied in this paper are identical to the ones used in [1] and [34].

\section{2) Results and Discussion}

a) Previous volumetric and shape findings: In a previously published analysis of the caudate nucleus, parcellated into anterior and posterior regions, repeated measures analysis of covariance (ANCOVA) with age as covariate, group as the between-subject factor and laterality or laterality plus region as the within-subject factor, revealed a significant group difference in total relative (corrected for brain size) volumes $(p=0.01)$. Followup Student's $t$ tests showed that right $(p<0.03)$ and left $(p<0.04)$ caudate nucleus total relative volumes were significantly smaller in subjects with schizotypal personality disorder than in normal comparison subjects. However, Student's t tests did not yield significant differences for the left $(p<0.06)$ or right $(p<0.2)$ anterior caudate relative volume but did yield a significant difference for the right $(p<0.04)$ but not for the left $(p=0.2)$ posterior caudate relative volume, [34]. 
In a recent analysis of the shape of the anterior caudate nucleus, repeated measures ANCOVA with age as covariate, group as the between-subject factor and laterality as the within-subject factor, revealed no main effect for diagnosis but a main effect for side and a significant interaction between side and diagnosis. Followup planned Student's $t$ tests showed a significant group difference for the right $(p=0.049)$ but not the left $(p=0.79)$ anterior caudate nucleus Shape Index measure [1].

The reported $p$ values for the followup $t$ tests were not corrected for multiple comparisons.

b) Shape characteristic findings: We applied our method to the anterior part of the caudate to investigate any possible difference in the shape between normal controls and SPD subjects. For each subject, the solution to Poisson's equation and its corresponding displacement map were computed for both left and right anterior caudate, as described in Section II. Shape characteristics were computed and our permutation test (Section II-D) showed a significant shape difference in the anterior right caudate $(p=0.023)$. A mean/std plot of the full feature vector is shown in Fig. 7. No significant difference was found on the left side ( $p=0.065$ ). The number of permutation used to establish the level of significance was $N=10000$. We are pleased to report that our method corroborates the shape findings, suggesting shape information may be able to find differences not otherwise detected by volume. Of note, the shape characteristic seems to have greater discriminatory power than the shape index measure. In fact, after Bonferroni correction for the shape characteristic tests only (corrected a significance level is 0.025), the difference on the left side is still significant.

\section{B. Premature Infants}

A full term baby is born after 40 weeks of gestation. In the United States, $12 \%$ of newborns are born prematurely each year. Recent reports have demonstrated an increase in cerebral palsy (a disorder of posture and movement) among children due to increased survival of premature infants born at less than 32 weeks gestational age and weighing less than 1500 grams. In fact, over 50\% of such very low birthweight infants will develop school difficulties (cognitive and behavioral), and about $10 \%$ will manifest cerebral palsy. The motor control and cognitive difficulties of these premature infants have long been thought to be related to white matter injury (WMI) [35]. Surprisingly, quantitative MRI studies have demonstrated that children born prematurely have evidence of predominantly cortical and subcortical gray matter abnormalities [36]-[39]. Since these gray matter structures are essential to cognitive function, it is hypothesized that these abnormalities may underlie the frequent cognitive impairments. One study of adolescents born prematurely showed that lower IQ scores correlated with smaller right caudate and left hippocampal nuclei [38]. Quantification of changes in these structures during the neonatal period will improve our understanding of the complex impact of early acquired injury on various brain structures and improve our ability to predict neurodevelopmental outcome. In this work, we applied our method for shape analysis to compare the shape of caudate nuclei of premature infants with and without asymmetric WMI. Our preliminary results demonstrate the ability of our method to find differences in the shape of such a small structure between the two groups of newborn infants.

1) Data-Thirteen premature infants with asymmetric WMI and eight premature infants without evidence of WMI (no injury, NI) underwent MRI scans at term equivalent age (3942 weeks postconceptional age, PCA). All infants were born at 24-29 weeks gestational age, but infants with WMI had germinal matrix-intraventricular hemorrhage and a unilateral periventricular hemorrhagic infarction (WMI). MRI data were acquired in the coronal plane with a 1.5T GE scanner, using a 3-D SPoiled Gradient Recalled (3-D SPGR) sequence 
yielding a voxel size of $0.703125 \times 0.703125 \times 1.5 \mathrm{~mm}$, as shown in Fig. 9. An expert user segmented the anterior part of the caudate nuclei (head of the caudate) manually using the 3D SPGR images. Details of the technique of manual segmentation have been previously described [34].

\section{2) Results and Discussion}

a) Volumetric findings: To investigate the difference in volumes in the anterior caudates between the infants with and without WMI in the two groups, we first normalized their volumes to intracranial cavity volume (ICC) and then performed a four-group (WMI injured side, WMI unaffected side, NI left side and NI right side) one way ANOVA, which revealed a main group effect $(p<0.0005)$. Post-hoc Tukey HSD tests (corrected for multiple comparisons), revealed a significant group difference between the WMI injured side and unaffected WMI $(p=0.001)$, left NI $(p<0.0005)$ and right NI $(p<0.0005)$. We found no difference between left and right NI $(p=0.913)$ and no difference between unaffected WMI and left $(p=0.526)$ or right $(p=0.168)$ NI sides.

b) Shape findings: Next, we applied our novel method to investigate whether there was any difference in the shape of the caudate head between the two groups of infants, and between sides. The caudate of an infant is much smaller than that of an adult and the segmented caudate nuclei are very coarse; thus, we used trilinear interpolation to increase the resolution of the segmented data to $0.5 \times 0.5 \times 0.5 \mathrm{~mm}$. Our permutation test $(N=10000$ permutations) revealed a statistically significant difference in the shape of the injured side in infants with WMI when compared with the contralateral side $(p=0.031)$. A mean/std plot of the full feature vector is shown in Fig. 10. We also found a statistically significant difference in the shape of the injured caudate head of the WMI group when compared with either caudate head of the NI group $(p<0.011)$, see Fig. 11. Of note, we found a statistically significant difference in shape between the unaffected side of the WMI group and either caudate of the NI group ( $p<0.033)$, as shown in Fig. 12. No significant difference in the shape of the caudate head was found between the left and right sides in the NI group $(p>0.05)$. The reported $p$ values were not corrected for multiple comparisons, the new significance level using Bonferroni method is 0.0125 , a very stringent threshold considering our sample size. Nevertheless, the difference in shape between affected WMI and either NI sides remains significant, even after this strict correction.

Hence, we were able to show significant differences in the shape of the head of the caudate nucleus, not only in the nuclei obviously affected by an ipsilateral hemorrhagic infarction, but also in the contralateral caudate in infants with WMI. These findings demonstrate the discriminative power of the method to detect changes in the shape of a structure, even when there is no difference in the volume when compared with normal controls. This technique will likely help elucidate the often subtle anatomical changes in structures affected directly or even at a distance by disease processes such as ischemic brain injury.

\section{CONCLUSION}

In this paper, we have presented a novel method to analyze the shape of anatomical structures. Our method is based on studying the solution of Poisson's equation and its associated displacement map to assess the dynamics of change of the equipotential surfaces as the potential increases inside the structure. We model this process by a simple function, called the shape characteristic, which expresses shape as "complexity $=f$ (potential)." The complexity is expressed as the coefficient of variance of the displacement along the equipotential set. This function is illustrated on 2-D synthetic and natural silhouettes, and we 
suggest that the shape characteristic could be used to automatically classify objects into different shape classes.

The main objective of this project was to compare the shape of an anatomical structure between different populations. We performed two neuroimaging studies, one on SPD for which we already had well-established volume and shape differences, and one on infants with WMI with no prior knowledge of the potential shape differences. Our results on the shape of the caudate nucleus in SPD correlated well with our previously reported finding [1], using an entirely different methodology, that the shape of the anterior portion of right caudate nucleus differed between subjects with SPD and NCs. This suggests that our method can be used as a powerful tool to correlate the shape of anatomical structures with different factors such as aging or disease type. We further illustrated the potential advantage of our approach by studying the effect of early acquired injury on the caudate nucleus in premature infants. Our technique not only confirmed the volumetric findings but also detected differences in shape not detected by the volumetric analysis. Namely, even though the caudate nucleus on the unaffected side of infants with white matter injury is not different in size than the caudate of normal infants, its shape is.

The "shape characteristic" is one of many tools one can build based upon Poisson's equation. It can certainly be improved, and we hope that our work will inspire others to further explore its potential for shape analysis. Nevertheless, our particular technique is already quite useful as it allows us to perform very simple statistical tests that are easy to understand and interpret. Unfortunately, the precise nature and location of the shape difference cannot be expressed with the "shape characteristic" due to its global nature. In future work, we propose to investigate how one can derive more local and intuitive representations from this equation and its solution and at the same time keep the statistical analysis simple and easily interpretable.

\section{Acknowledgments}

The work of H. Haidar was supported by the William Randolph Hearst Foundation. The work of R. W. McCarley was supported in part by the National Institutes of Health under Grant R01-MH052807 and Grant R01-MH40799, and in part by the Department of Veterans Affairs Merit Awards. The work of M. E. Shenton was supported in part by the National Institutes of Health under Grant K05-MH070047, Grant R01-MH50747, and Grant U54-EB005149, and in part by the Department of Veterans Affairs Merit Awards. The work of J. S. Soul was supported in part by the National Institutes of Health under Grants P01-NS38475 and MRDDRC P30-HD18655, and in part by the United Cerebral Palsy Foundation.

\section{REFERENCES}

1. Levitt JJ, Westin C-F, Nestor PG, San-Jose R, Dickey CC, Voglmaier MM, Seidman LJ, Kikinis R, Jolesz FA, McCarley RW, Shenton ME. Shape of the caudate nucleus and its cognitive correlates in neuroleptic-naive schizotypal personality disorder. Biological Psychiatry. 2004; vol. 55:177-184. [PubMed: 14732598]

2. Thomson PM, Miller MI, Ratnather T, Poldrack RA, Nichols TE. Mathematics in brain imaging. NeuroImage. 2004; vol. 23

3. Brechbühler C, Gerig G, Kübler O. Parametrization of closed surfaces for 3-d shape description. Comput. Vision Image Understand. 1995; vol. 61:154-170.

4. Mangin, JF.; Poupon, F.; Riviere, D.; Cachia, A.; Collins, DL.; Evans, A.; Regis, J. 3-D moment invariant based morphometry. In: Ellis, RE.; Peters, TM., editors. Proc. MICCAI ser. LNCS. Vol. vol. II. 2003. p. 505-512.

5. Cootes T, Taylor CJ, Cooper DH, Graham J. Active shape models-their training and applications. Comput. Vision Image Understand. 1995; vol. 61:38-59.

6. Bookstein FL. Shape and the information in medical images. Comput. Vision Image Understand. 1997; vol. 66(no. 2):97-118. 
7. Csernansky JG, Joshi S, Wang L, Haller JW, Gado M, Miller JP, Grenander U, Miller MI. Hippocampal morphometry in schizophrenia by high dimensional brain mapping. Proc. Nat. Acad. Sci. 1998; vol. 95(no. 19):11406-11411. [PubMed: 9736749]

8. Thompson PM, Hayashi KM, de Zubicaray GI, Janke AL, Rose SE, Semple J, Hong MS, Herman DD, Gravano D, Droddrell DM, Toga AW. Mapping hippocampal and ventricular change in Alzheimer disease. NeuroImage. 2004; vol. 22:1754-1766. [PubMed: 15275931]

9. Bookstein FL. Comments: "Voxel-based morphometry" should not be used with imperfectly registered images. NeuroImage. 2001; vol. 14(no. 6):1454-1462. [PubMed: 11707101]

10. Ashburner J, Friston KJ. Why voxel-based morphometry should be used. NeuroImage. 2001; vol. 14(no. 6):1238-1243. [PubMed: 11707080]

11. Hellier P, Barillot C, Corouge I, Gibaud B, Goualher GL, Collins DL, Evans A, Malandain G, Ayache N, Christensen GE, Johnson HJ. Retrospective evaluation of intersubject brain registration. IEEE Trans. Med. Imag. 2003 Sep; vol. 22(no. 9):1120-1130.

12. Golland P, Grimson WE, Shenton ME, Kikinis R. Detection and analysis of statistical differences in anatomical shape. Med. Image Anal. 2005; vol. 9(no. 1):69-86. [PubMed: 15581813]

13. Styner M, Lieberman J, Pantazis D, Gerig G. Boundary and medial shape analysis of the hippocampus in schizophrenia. Med. Image Anal. 2004; vol. 8:197-203. [PubMed: 15450215]

14. Bouix S, Pruessner JC, Collins DL, Siddiqi K. Hippocampal shape analysis using medial surfaces. NeuroImage. 2005; vol. 25(no. 4):1077-1089. [PubMed: 15850726]

15. Styner M, Gerig G, Lieberman J, Jones D, Weinberger D. Statistical shape analysis of neuroanatomical structures based on medial models. Med. Image Anal. 2003; vol. 7(no. 3):207220. [PubMed: 12946464]

16. Gorelick, L.; Galun, M.; Sharon, E.; Basri, R.; Brandt, A. Shape representation and classification using the Poisson equation; Proc. IEEE Conf. Comput. Vision Pattern Recognit; Washington, DC: 2004. p. 61-67.

17. Jones SE, Buchbinder BR, Aharon I. Three-dimensional mapping of cortical thickness using Laplace's equation. Hum. Brain Mapp. 2000; vol. 11:12-32. [PubMed: 10997850]

18. Yezzi AJ, Prince JL. An Eulerian PDE approach for computing tissue thickness. IEEE Trans. Med. Imag. 2003 Oct; vol. 22(no. 10):1332-1339.

19. Leventon, M.; Grimson, WEL.; Faugeras, O. Statistical shape influence in geodesic active contours; Proc. IEEE Conf. Comput. Vision Pattern Recognit; 2000. p. 316-323.

20. Tsai A, Wells WM, Warfield S, Wilsky AS. An EM algorithm for shape classification based on level sets. Med. Image Anal. 2005; vol. 9:491-502. [PubMed: 16046181]

21. Blum H. Biological shape and visual science. J. Theoretical Biol. 1973; vol. 38:205-287.

22. Pizer SM, Siddiqi K, Székeley G, Damon JN, Zucker SW. Multiscale medial axes and their properties. Int. J. Comput. Vision. 2003; vol. 55(no. 3)

23. Siddiqi K, Kimia BB, Tannenbaum AR, Zucker SW. Shock graphs and shape matching. Int. J. Comput. Vision. 1999; vol. 35(no. 1):13-32.

24. Lapidus, L.; Pinder, GF. Numerical Solutions of Partial Differential Equations in Science and Engineering. New York: Wiley; 1982.

25. Johnson, C. Numerical Solution of Partial Differential Equations by the Finite Element Method. Cambridge, U.K.: Cambridge Univ. Press; 1988.

26. Press, WH.; Teukolsky, SA.; Vetterling, WT.; Flannery, BP. Numerical Recipes. Cambridge, U.K.: Cambridge Univ. Press; 1992.

27. Haidar H, Egorova S, Soul JS. New numerical solution of the Laplace equation for tissue thickness measurement in three dimensional MRI. J. Math. Modelling Algorithms. 2005; vol. 4:83-97.

28. Duda, RO.; Hart, PE.; Stork, DG. Pattern Classification. 2nd ed.. New York: Wiley; 2000.

29. Good, P. Permutation, Parametric, and Bootstrap Tests of Hypotheses. 3rd ed.. New York: Springer; 2004.

30. Nichols TE, Holmes AP. Nonparametric tests for functional neuroimaging: A primer with examples. Hum. Brain Mapp. 2001; vol. 15:1-25. 
31. Bullmore ET, Stuckling J, Overmeyer S, Rabe-Hesketh S, Taylor E, Brammer MJ. Global, voxel, and cluster tests, by theory and permutation, for a difference between two groups of structural MR images of the brain. IEEE Trans. Med. Imag. 1999 Jan; vol. 18(no. 1):32-42.

32. Keshavan MS, Rosenberg D, Sweeney JA, Pettegrew JW. Decreased caudate volume in neuroleptic-naive psychotic patients. Amer. J. Psychiatry. 1998; vol. 155:774-448. [PubMed: 9619149]

33. Corson PW, Nopoulos P, Andreasen NC, Heckel D, Arndt S. Caudate size in first-episode neuroleptic-naive schizophrenic patients measured using an artificial neural network. Biological Psych. 1999; vol. 46(no. 5):712-720.

34. Levitt JJ, McCarley RW, Dickey CC, Voglmaier MM, Niznikiewicz MA, Seidman LJ, Hirayasu Y, Ciszewski AA, Kikinis R, Jolesz FA, Shenton ME. MRI study of caudate nucleus volume and its cognitive correlates in neuroleptic-naive patients with schizotypal personality disorder. Amer. J. Psychiatry. 2002; vol. 159:1190-1197. [PubMed: 12091198]

35. Volpe, J. Neurology of the Newborn. Philadelphia, PA: W.B. Saunders; 2001.

36. Inder TE, Huppi PS, Warfield SK, Kikinis R, Zientara GP, Barnes PP, Jolesz F, Volpe JJ. Periventricular white matter injury in the premature infant is followed by reduced cerebral cortical gray matter volume at term. Ann. Neurol. 1999; vol. 46(no. 5):755-760. [PubMed: 10553993]

37. Peterson BS, Vohr B, Staib LH, Dolberg A, Schneider KC, Katz KH, Westerveld M, Sparrow S, Anderson AW, Duncan CC, Makuch RW, Gore JC, Ment LR. Regional brain volume abnormalities and long-term cognitive outcome in preterm infants. J. Amer. Med. Assoc. 2000; vol. 284(no. 15):1939-1947.

38. Abernethy LJ, Palaniappan M, Cooke RW. Quantitative magnetic resonance imaging of the brain in survivors of very low birth weight. Arch. Dis. Child. 2002; vol. 87(no. 4):279-283. [PubMed: 12243993]

39. Inder T, Wells S, Mogridge N, Spencer C, Volpe J. Defining the nature of the cerebral abnormalities in the premature infant: A qualitative magnetic resonance imaging study. $\mathrm{J}$. Pediatrics. 2003; vol. 143(no. 2):171-179. 

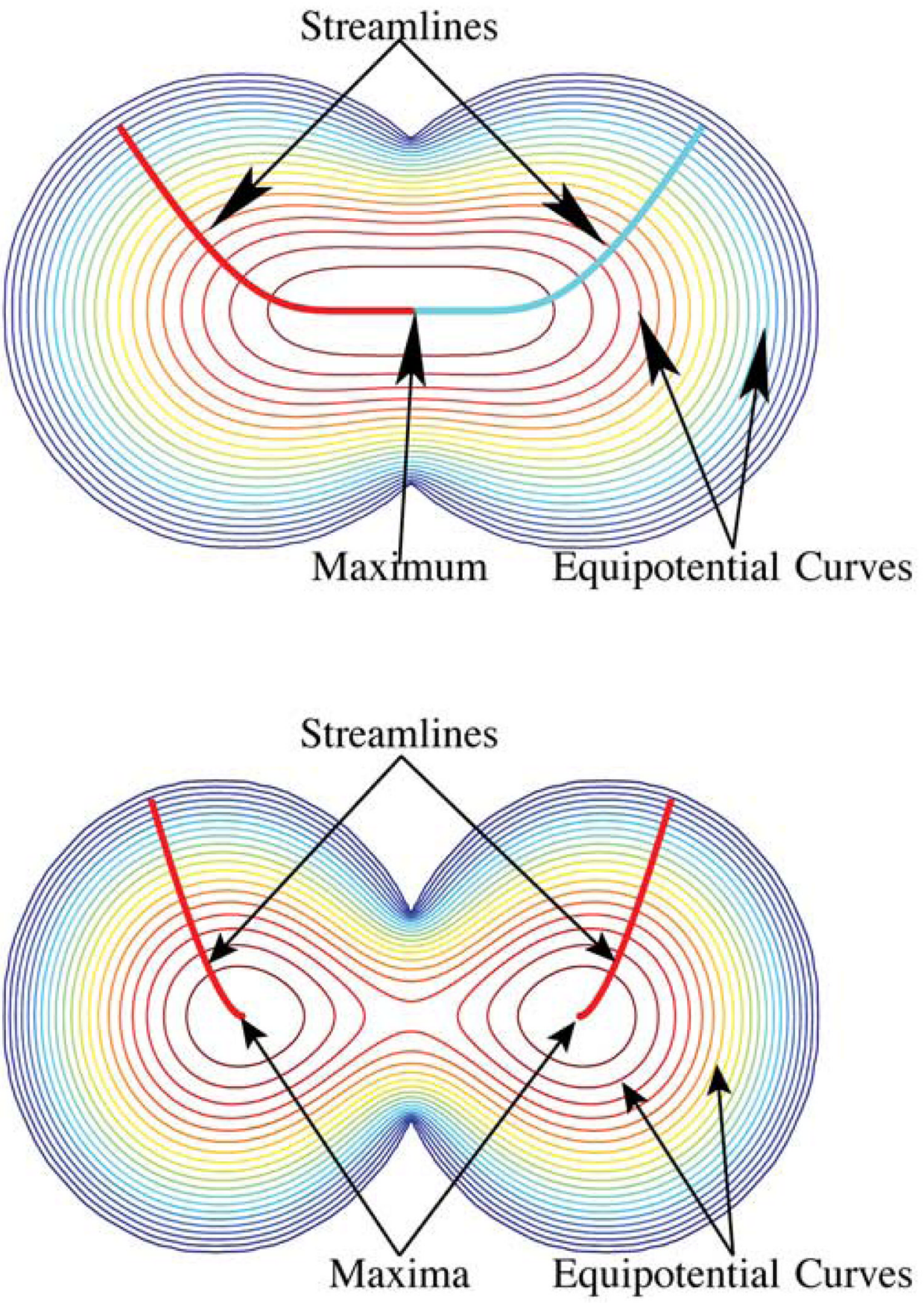

Fig. 1.

Solution to Poisson's equation for two different shapes. Top: Solution has only one maximum and all streamline converge to it. Bottom: Solution has two local maxima and the streamlines can converge to either one of them, suggesting a classification of the object into distinctive parts. 

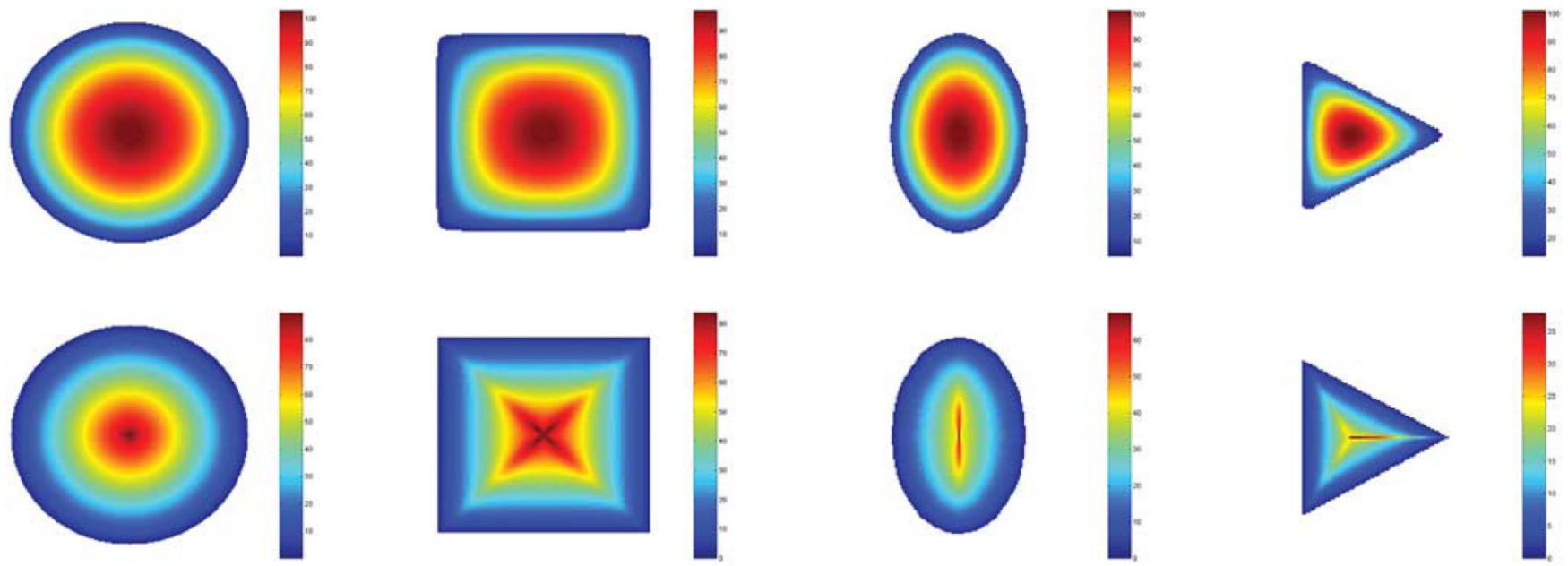

Fig. 2.

Top: Solution to Poisson's equation for a variety of 2-D synthetic shapes. Bottom: Corresponding displacement maps. 


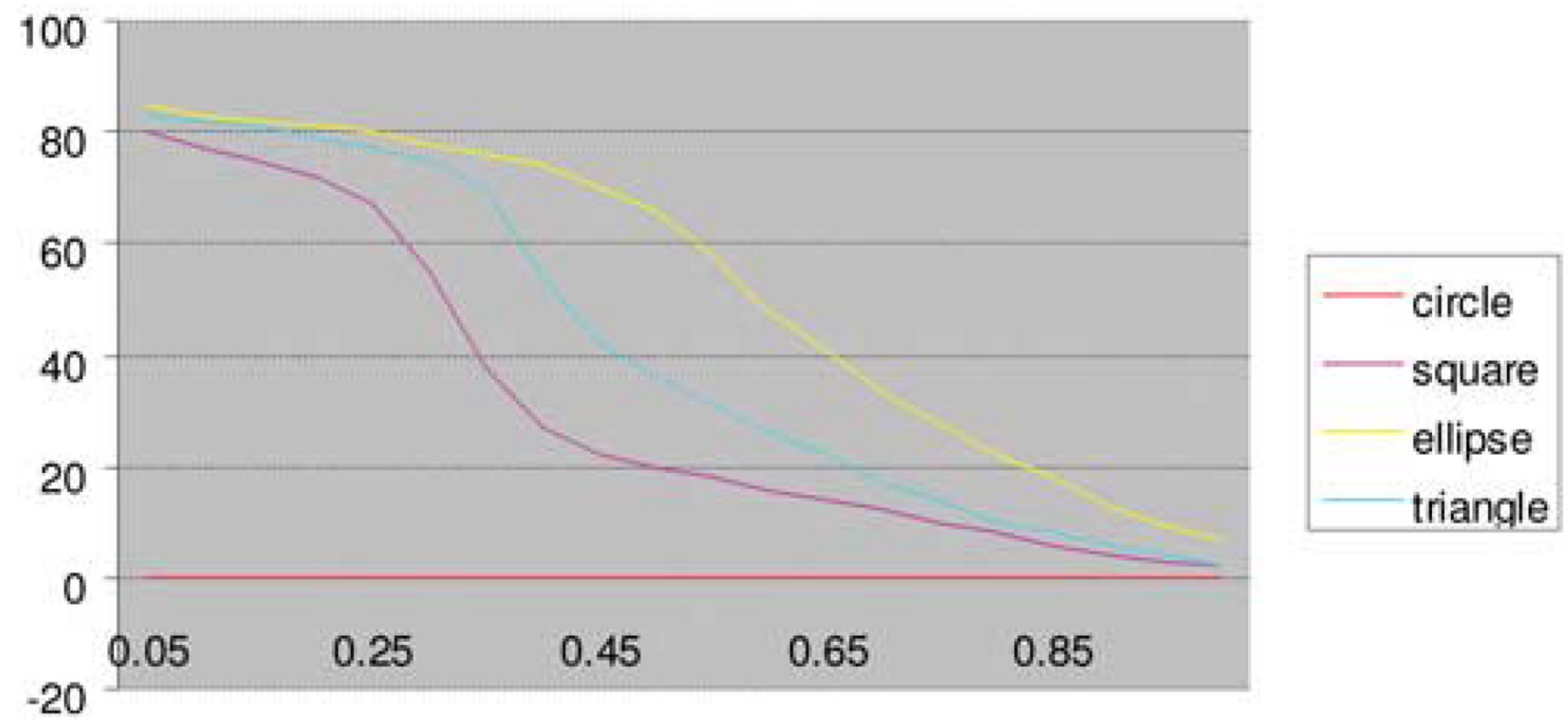

Fig. 3.

Shape characteristic curves of synthetic 2-D objects. 


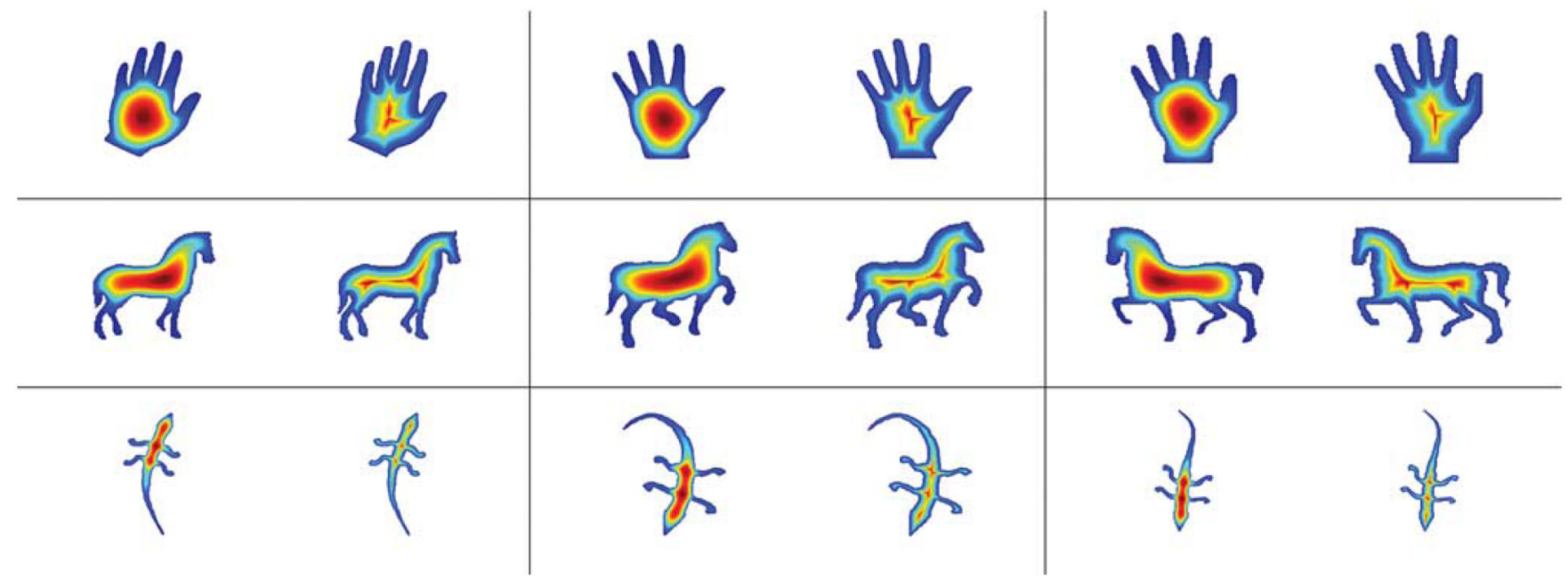

Fig. 4.

Example of solutions and displacement maps of Poisson's equation on natural 2-D silhouettes. In each cell, the solution to Poisson's equation is on the left and the displacement map on the right. 


\section{nu(E) for Natural Objects}

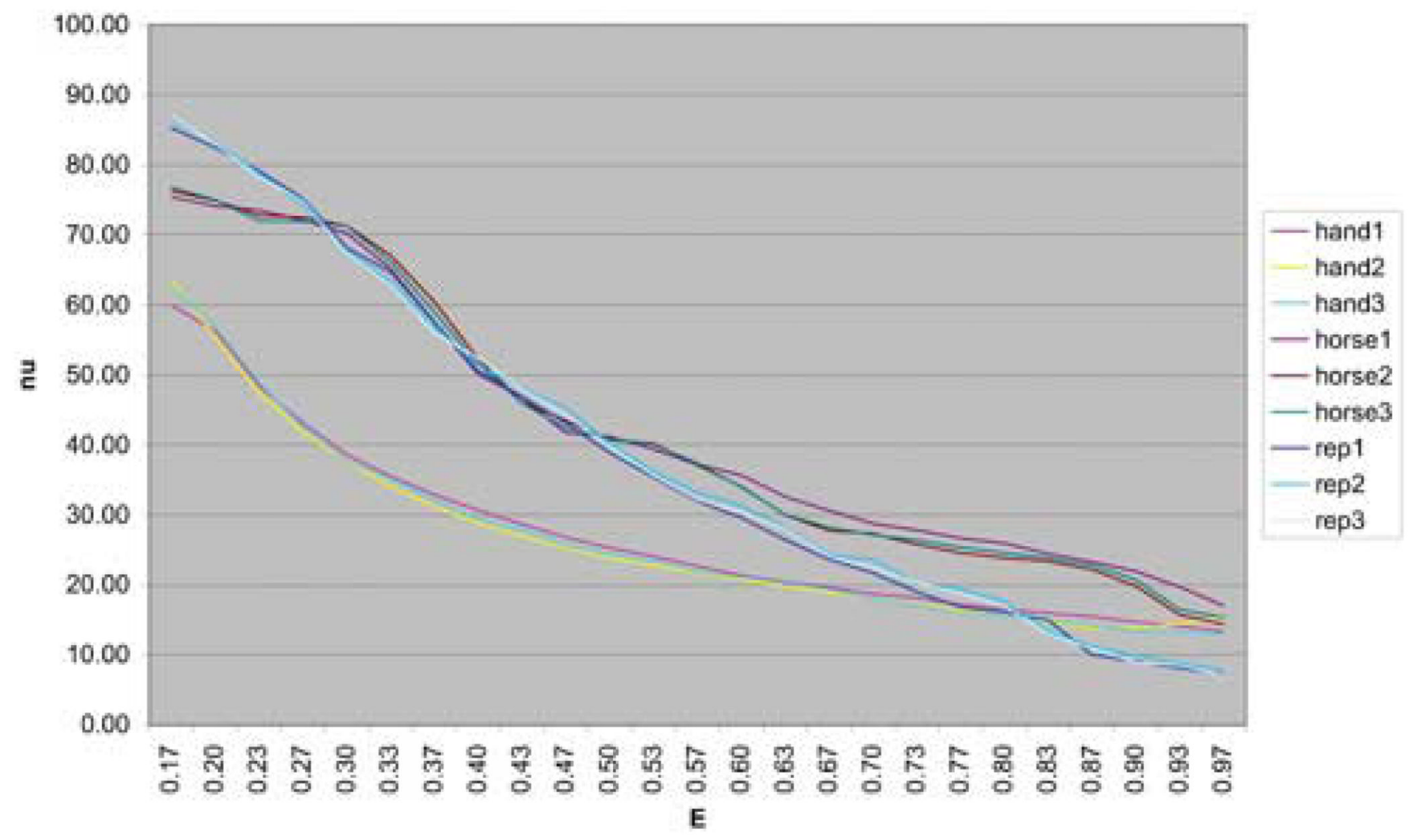

Fig. 5.

Shape characteristic curves of 2-D silhouettes of natural objects. 


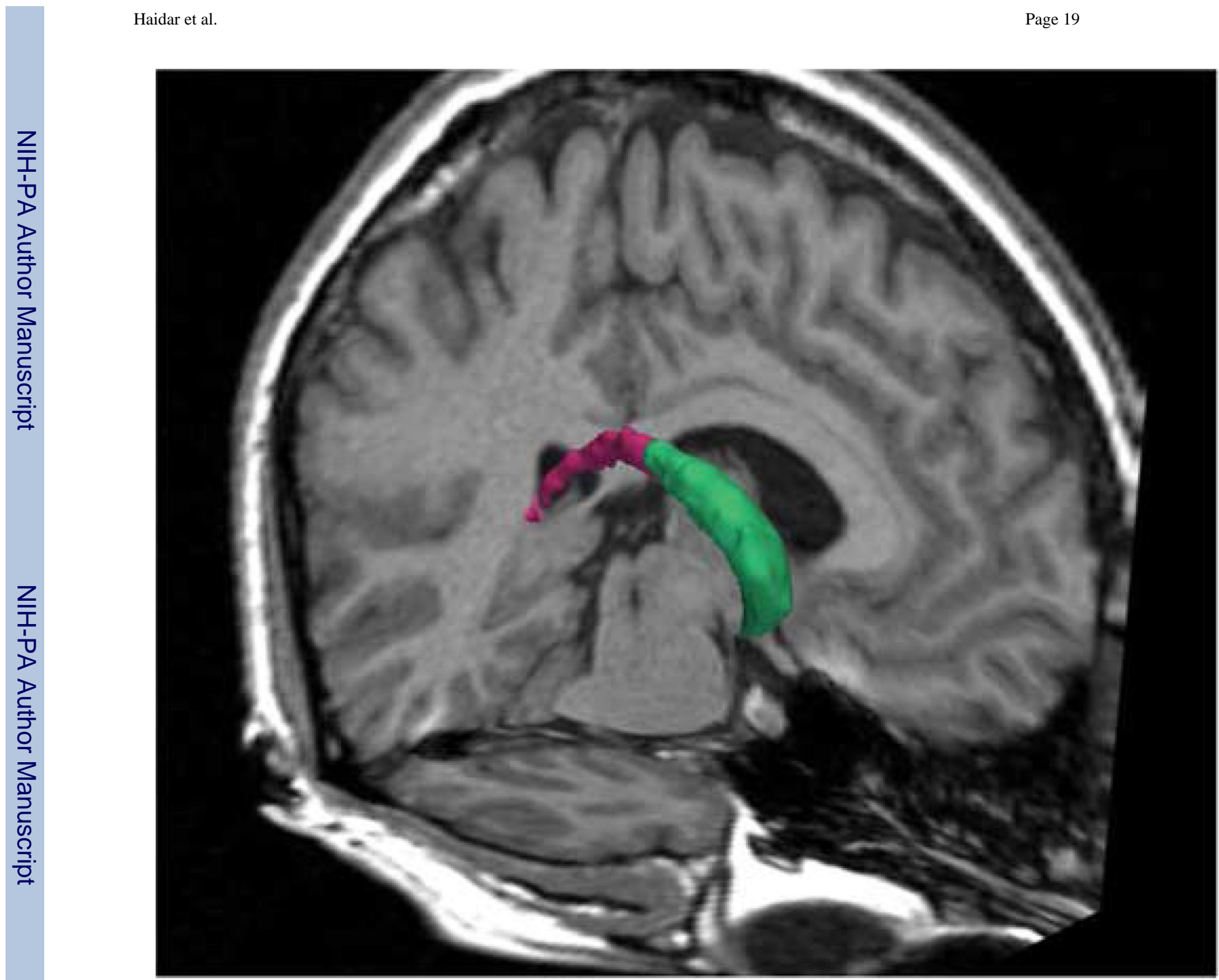

Fig. 6.

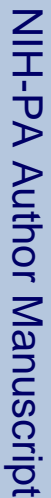

3-D rendering of the caudate nucleus in an adult brain. 


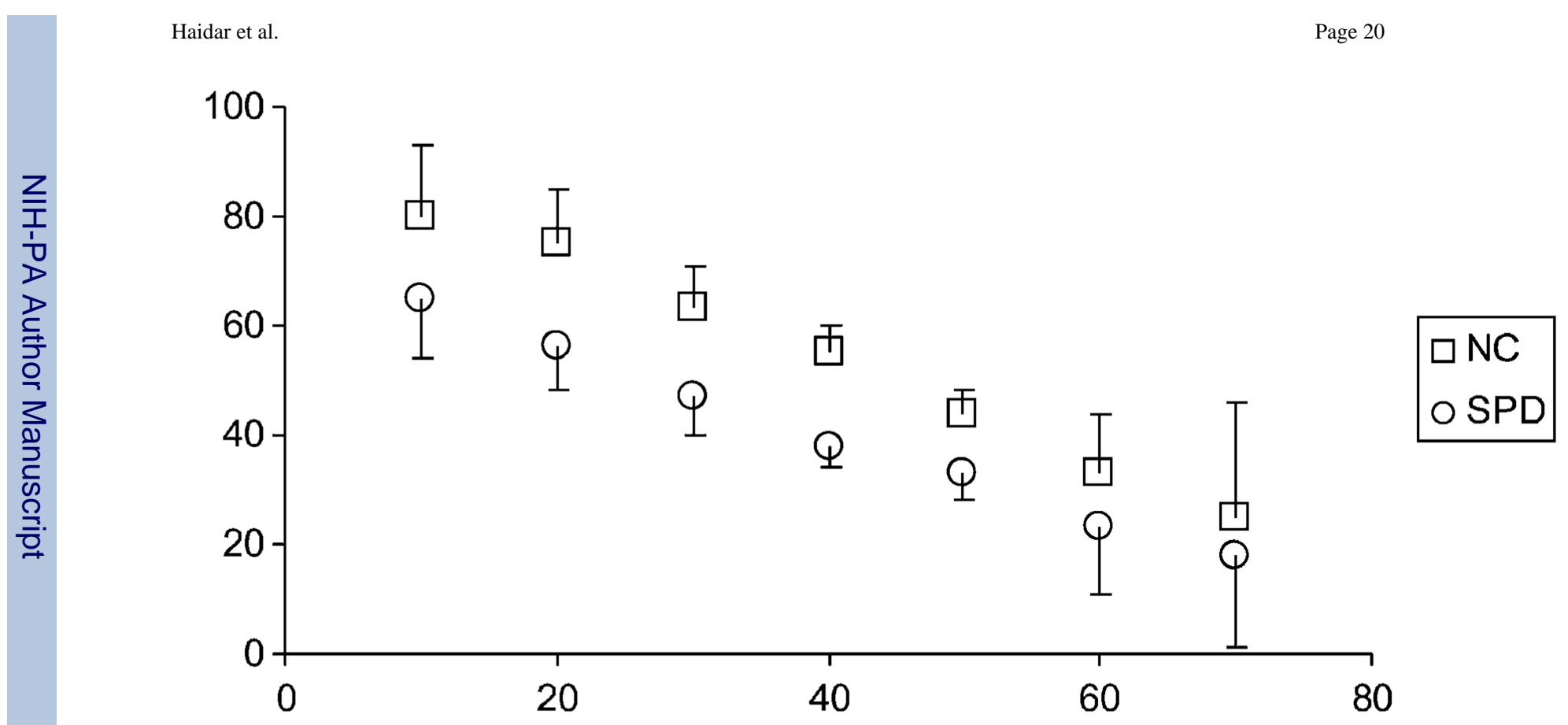

Fig. 7.

Shape characteristics of the anterior part of the right caudate nucleus in SPD and normal subjects. 

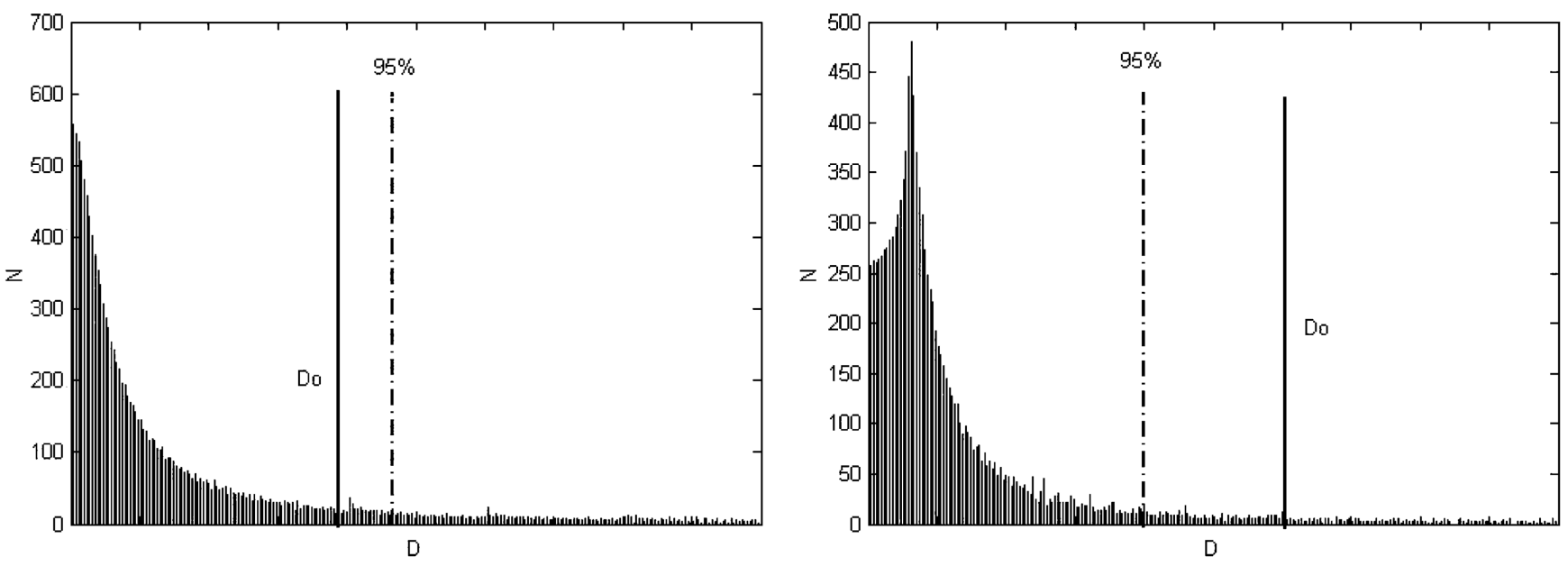

Fig. 8.

Sample distributions created through random permutations of the input data. $D_{0}$ corresponds to the difference associated with the original label. Left: Differences of the mean of the shape characteristics of the left anterior caudate nuclei between SPD and NC. Right: Differences of shape characteristic mean of the right anterior caudate between SPD and NC. 


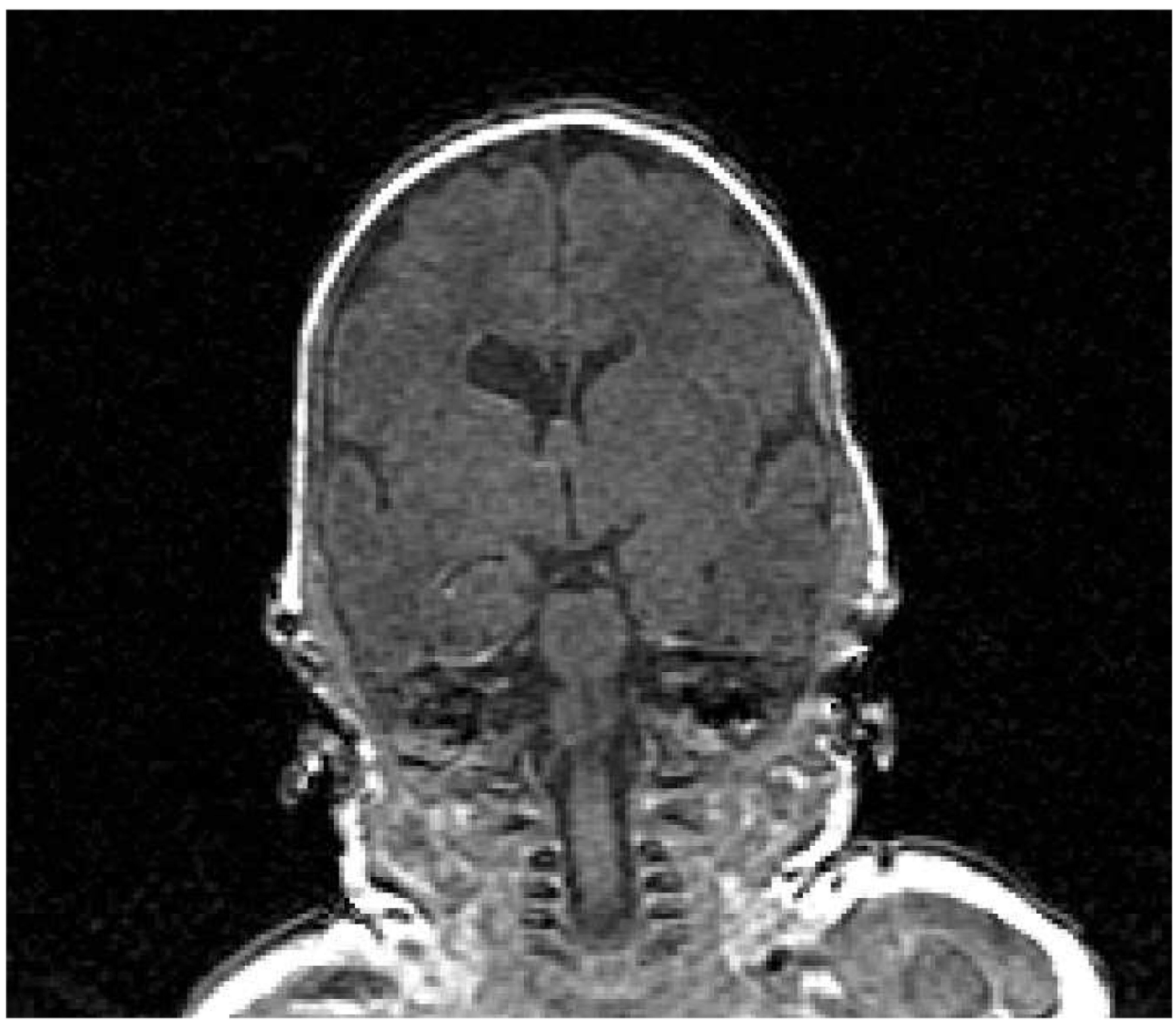

Fig. 9.

Coronal slice of an SPGR scan of a premature infant with unilateral white matter injury. 


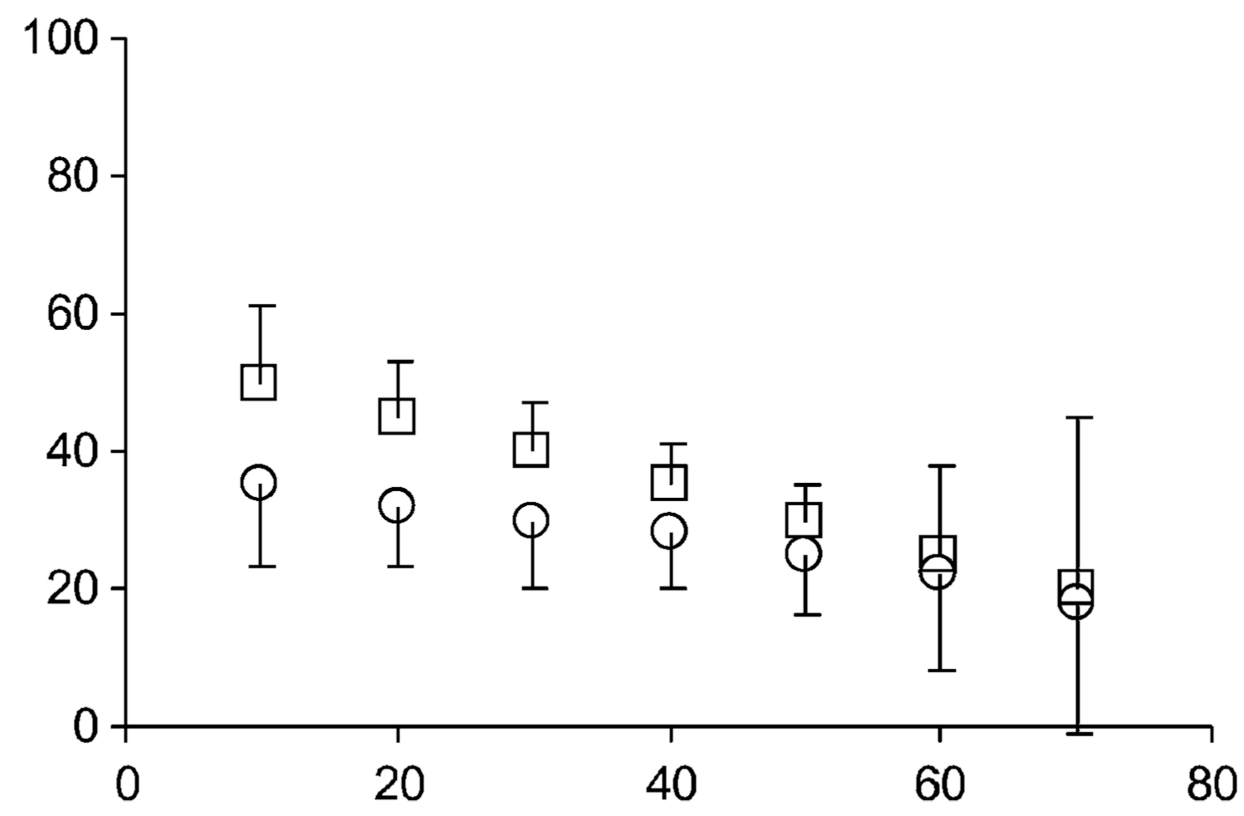

Fig. 10.

Shape characteristics of anterior part of the caudate nucleus in injured and healthy sides in WMI. 


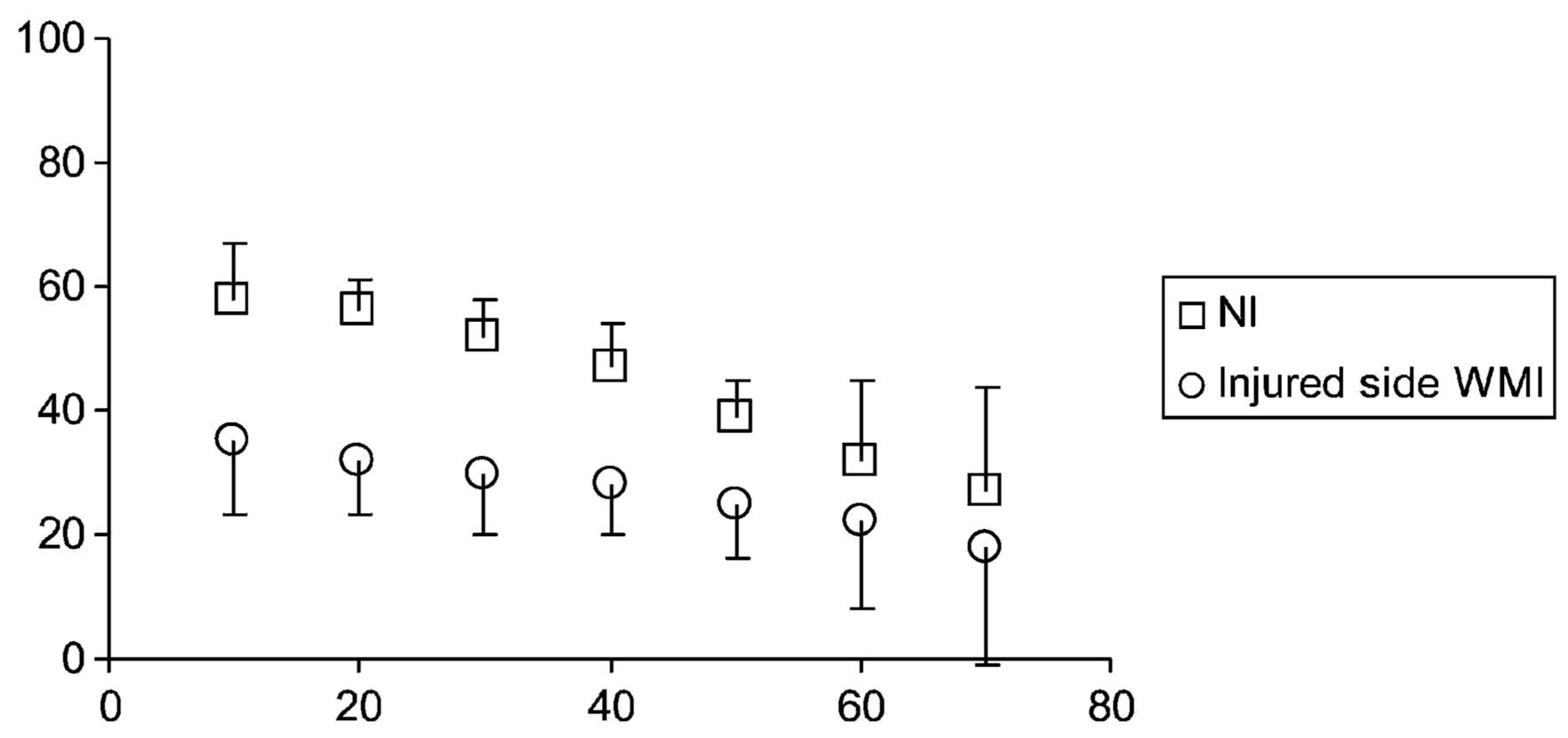

Fig. 11.

Shape characteristics of the anterior part of the caudate nucleus in NI infants and affected side of WMI subjects. 


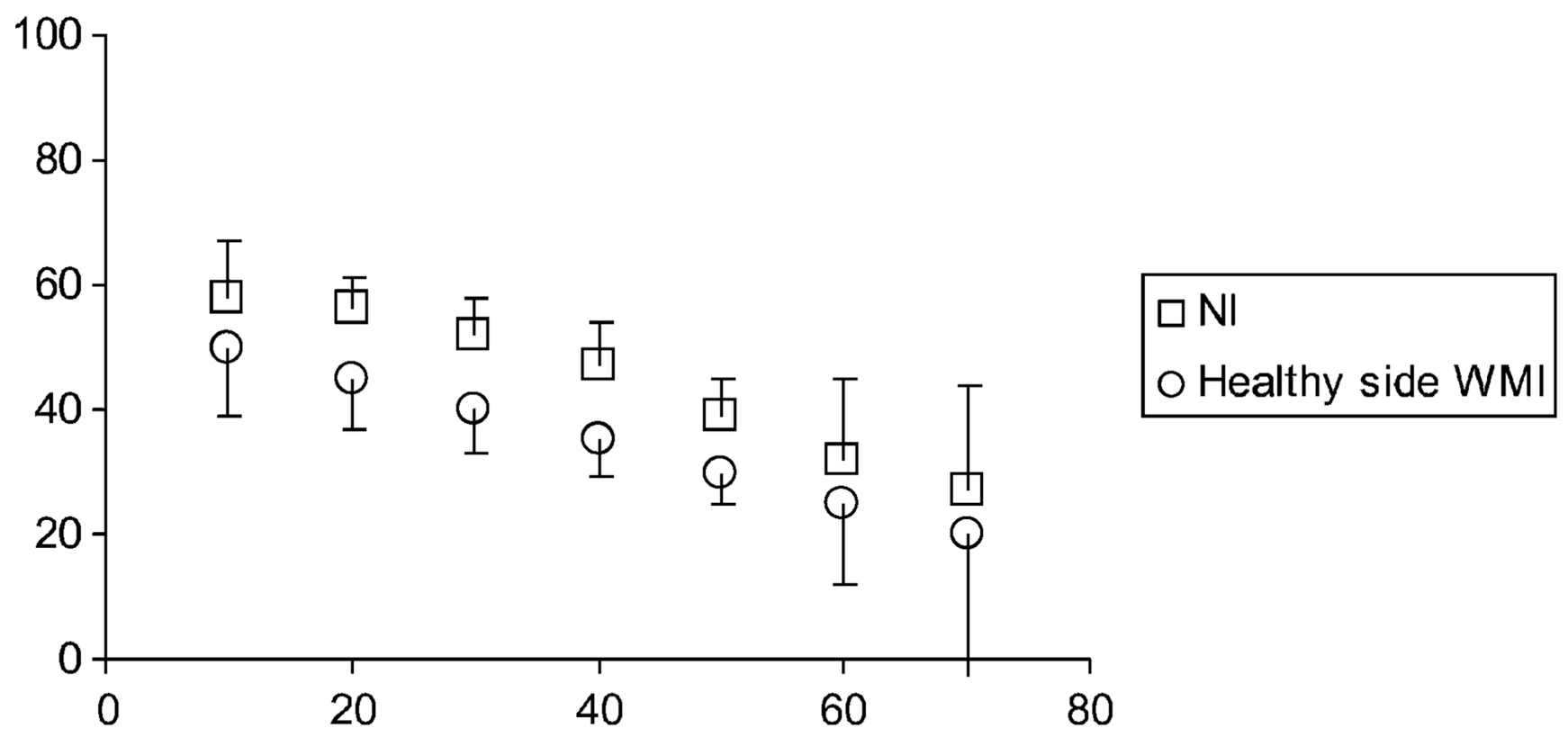

Fig. 12.

Shape characteristics of the anterior part of the caudate nucleus in NI infants and healthy side of WMI subjects. 